\title{
Effect of silicone oil on macular capillary vessel density and thickness
}

\author{
WU XIANG, YANTAO WEI, WEI CHI, ZHAOTIAN ZHANG, LITING ZHONG, \\ RONGJIAO LIU and SHAOCHONG ZHANG
}

State Key Laboratory of Ophthalmology, Zhongshan Ophthalmic Center, Sun Yat-sen University, Guangzhou, Guangdong 510060, P.R. China

Received April 7, 2019; Accepted September 19, 2019

DOI: $10.3892 /$ etm.2019.8243

\begin{abstract}
The present study assessed changes in macular capillary vessel density and thickness associated with ocular silicone oil tamponade (SOT) following complex vitreoretinal surgery. A total of 23 patients who underwent pars plana vitrectomy combined with intravitreal SOT were retrospectively enrolled, and a single eye was assessed in each participant. Furthermore, 20 patients who required silicone oil removal (SOR) were included. The macular capillary vessel density and the foveal avascular zone (FAZ) area were analyzed using optical coherence tomography angiography (OCTA) and retinal thickness was assessed by OCT. The results demonstrated that the macular capillary vessel density of the superficial capillary plexus (SCP) and deep capillary plexus (DCP), the FAZ area and full retinal thickness were retained at a stable level following SOT $(\mathrm{P}>0.05)$, and also following $\mathrm{SOR}(\mathrm{P}>0.05)$. Furthermore, the parafoveal $(\mathrm{P}=0.008)$, superior-hemi $(\mathrm{P}=0.007)$, temporal $(\mathrm{P}=0.015)$, superior $(\mathrm{P}=0.028)$ and nasal $(\mathrm{P}=0.002)$ inner retinal thickness decreased in the SOT group, whereas the inner retinal thickness was unaltered after SOR vs. baseline $(\mathrm{P}>0.05)$. In conclusion, silicone oil has no significant effect on macular capillary vessel density within a 6-month period but may compress and reduce the thickness of the inner retina.
\end{abstract}

\section{Introduction}

Silicone oils are constituted of a linear chain of siloxane repeat units (-Si-O) and are considered to be an ideal substitute material for the vitreous. Silicone oil has been widely used in complex vitreoretinal surgery since Cibis et al (1) first

Correspondence to: Professor Shaochong Zhang, State Key Laboratory of Ophthalmology, Zhongshan Ophthalmic Center, Sun Yat-sen University, 54S Xianlie Road, Guangzhou, Guangdong 510060, P.R. China

E-mail: zhangshaochong@gzzoc.com

Key words: silicone oil, macular capillary vessel density, retinal thickness, optical coherence tomography angiography demonstrated the intravitreal use of silicone oil in 1962, and is an important auxiliary tool in complex vitreoretinal surgery. The common indications for silicone oil tamponade (SOT) are proliferative vitreoretinopathy, severe diabetic retinopathy, large retinal tears, viral retinitis and ocular trauma $(2,3)$. The success rate of vitreoretinal surgery has markedly increased since the introduction of SOT and the range of applications has expanded to the treatment of macular holes in highly myopic eyes (4), chronic and persistent macular holes, colobomatous retinal detachment (5) and chronic uveitis with hypotony (6). However, the long-term use of intraocular SOT is thought to cause complications, including cataracts, keratopathy and glaucoma. Therefore, silicone oil is commonly used in temporary intraocular tamponade and is recommended to be removed as soon as possible (7). However, there is no consensus on when to remove the silicone oil and most surgeons do so between 3 and 6 months to avoid alleged late complications, including optic atrophy from glaucoma or tissue impregnation (8).

Optical coherence tomography angiography (OCTA) is a novel non-invasive vascular imaging technique used to display retinal and choroidal blood vessels, providing a novel method for the assessment of associated vascular diseases $(9,10)$. Intraocular silicone oil has been commonly used as a vitreous substitute for $>5$ decades. However, whether silicone oil is toxic to the retinal vasculature and whether the compressing effect of silicone oil decreases retinal thickness has remained to be elucidated. To address these questions, the association between silicone oil treatment and the condition of the retinal vessels was explored using OCTA. In addition, retinal thickness was evaluated in patients with SOT using OCT.

\section{Materials and methods}

Study participants. The present study retrospectively analyzed the results of 23 patients with complex vitreoretinal disease, reported at Zhongshan Ophthalmic Center (Sun Yat-sen University, Guangzhou, China) between July 2016 and September 2017, and a single eye was assessed in each participant. These patients had previously received pars plana vitrectomy, laser coagulation and SOT, had used perfluorocarbon liquids where necessary (SOT group) and had all received OCTA examinations $\sim 1$ and 3 months into the follow-up period. Furthermore, 20 patients were included 
who had received SOT between January 2016 and April 2017, had required silicone oil removal (SOR group), and who had received an OCTA examination $\sim 1$ week previously, as well as 3 months after SOR. Patients with glaucoma, keratopathy, severe cataracts, a re-detached retina, diabetes or any other severe systemic diseases were excluded from the study. All patients underwent comprehensive ocular examinations, including best-corrected visual acuity, intraocular pressure, slit lamp biomicroscopy and dilated fundus examinations. OCT and OCTA images with correct segmentation and without macular edema and artifacts were included, and those with incorrect segmentation, masking, black bands, bright lines and distorted stripes following en face OCTA were excluded. The study was performed in accordance with the Declaration of Helsinki and with the approval of the Ethics Committee of Zhongshan Ophthalmic Center (Guangzhou, China). Informed surgical consent was obtained from each participant.

OCTA. OCT and OCTA were performed using a spectral domain system (RTVue-XR Avanti; Optovue), a device with a high speed of 70,000 axial scans per second, using a light source with a wavelength of $840 \mathrm{~nm}$ and with an axial resolution of $5 \mu \mathrm{m}$. The AngioVue OCTA system is based on a split-spectrum amplitude decorrelation angiography algorithm, using blood flow as an intrinsic contrast (11). In the present study, each patient underwent cross-line, retina map and angio-retinal scanning. All procedures were performed using RTVue-XR Avanti software (version 2016.2.0.35; Optovue), where the vessel output of the OCTA algorithm is an image of the retinal and choroidal vasculature, which may be segmented into four zones: The superficial capillary plexus, deep capillary plexus, outer retina and choroid (12).

Assessment of the foveal avascular zone (FAZ) area and retinal vasculature. In the present study, $3 \times 3-\mathrm{mm}$ macular OCTA scans were acquired. The FAZ area, foveal and parafoveal vessel densities of the retinal vasculature, which incorporates the superficial capillary plexus (SCP) and deep capillary plexus (DCP), were evaluated using the integrated analytics software of the AngioVue device. The foveal area was defined as a circle with a 1-mm diameter, centered on the fovea; the parafoveal area was defined as an annulus with an outer diameter of $3 \mathrm{~mm}$ and an inner diameter of $1 \mathrm{~mm}$ centered on the fovea. Furthermore, the parafovea was divided into four parts: Tempo, superior, nasal and inferior. The foveal and parafoveal vessel densities were defined as the percentage area occupied by vessels in the corresponding segmented areas.

Retinal thickness. Retinal thickness was determined using the retinal map protocol, which calculates the full and inner retinal thickness. The full retinal thickness was defined as the distance between the internal limiting membrane and the center of the retinal pigment epithelial cell (RPE), and the inner retinal thickness, between the internal limiting membrane and the outer boundary of the inner plexiform layer. The full and inner retinal thicknesses of the foveal and parafoveal areas were automatically calculated using the system's software and were defined as the mean thicknesses of each area (13).
Table I. Demographic data and clinical characteristics of the subjects.

\begin{tabular}{lcc}
\hline Variable & SOT $(\mathrm{n}=23)$ & SOR $(\mathrm{n}=20)$ \\
\hline Age (years) & $46.57 \pm 15.64$ & $47.00 \pm 16.68$ \\
Pre-operative BCVA & $0.12 \pm 0.21$ & $0.08 \pm 0.07$ \\
Final BCVA & $0.18 \pm 0.22$ & $0.13 \pm 0.16$ \\
Eyes with macular-off RRD & $22(95.65)$ & $20(100)$ \\
Duration of silicone & $5.56 \pm 2.17$ & $7.35 \pm 3.07$ \\
oil application (months) & & \\
\hline
\end{tabular}

Values are expressed as the mean \pm standard deviation or $\mathrm{n}(\%)$. BCVA, best corrected visual acuity; macula-off, retinal detachment involving the macula; RRD, rhegmatogenous retinal detachment; SOR, silicone oil removal group; SOT, silicone oil tamponade group.

Statistical analysis. Statistical analyses were performed using SPSS software version 13 (SPSS Inc.). A paired t-test was used to compare the capillary vessel densities, FAZ area and retinal thickness between the baseline and follow-up measurements. The statistical analyses were two-tailed and $\mathrm{P}<0.05$ was considered to indicate a statistically significant difference.

\section{Results}

Demographic and clinical features. A total of 23 patients who underwent vitreoretinal surgery with SOT were recruited. The participants in the SOT group included 17 males and 6 females, and a single eye was assessed in each participant; the mean age was $46.57 \pm 15.64$ years (range, $14-67$ years). In addition, 20 patients were recruited in the SOR group. Participants in the SOR group included 15 males and 5 females, with a mean age of $47.00 \pm 16.68$ years (range, $8-68$ years). The mean duration of SOT in the SOT group was 5.56 \pm 2.17 months. The demographic and clinical features of the subjects are listed in Tables I and SI, and representative images of the retinal capillary density measurement and FAZ area are provided in Fig. 1.

Macular capillary vessel density and thickness. In reference to the $3 \times 3-\mathrm{mm}$ macular OCTA images, the mean capillary density of the SCP and DCP, FAZ area and full retinal thickness remained at a stable level in the SOT $(\mathrm{P}>0.05)$ and SOR $(\mathrm{P}>0.05)$ groups. The inferior inner retinal thickness remained unchanged, whereas the parafoveal $(\mathrm{P}=0.008)$, superior-hemi $(\mathrm{P}=0.007)$, temporal $(\mathrm{P}=0.015)$, superior $(\mathrm{P}=0.028)$ and nasal $(\mathrm{P}=0.002)$ inner retinal thickness was decreased in the SOT group. Furthermore, the inner retinal thickness was unaltered in the SOR group ( $\mathrm{P}>0.05$; Tables II-V).

\section{Discussion}

In the present study, OCT and OCTA were used to evaluate the FAZ area, the vessel density of the retinal capillary plexuses and the retinal thickness in the eyes of patients with SOT. The results indicated that the FAZ area, SCP and DCP vessel densities and the full retinal thickness were not altered by SOT or following SOR. The inner retinal thickness remained stable prior to and 

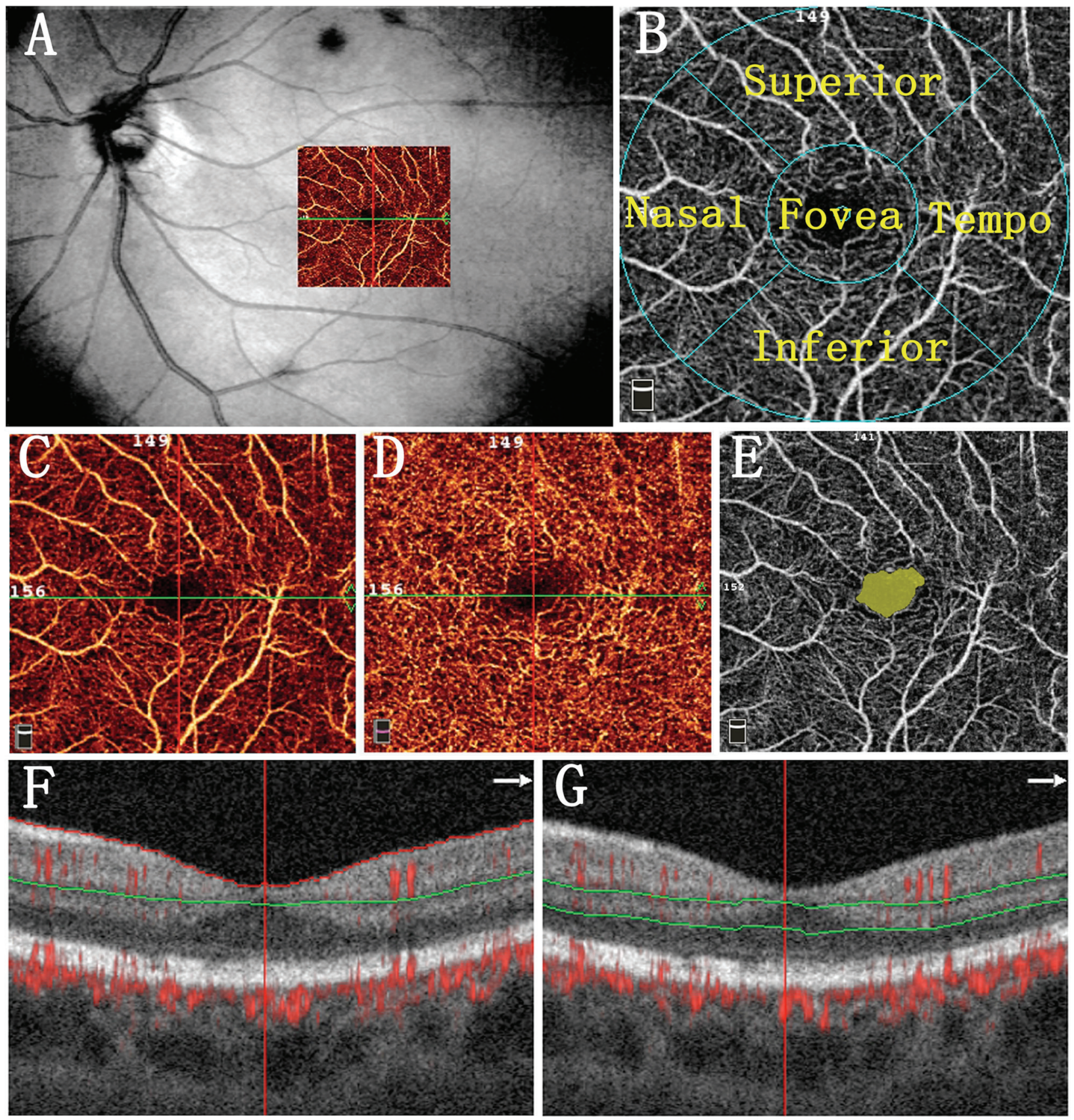

Figure 1. OCTA en face maps of the left eye of a patient with silicone oil tamponade. (A) The small, $3 \times 3 \mathrm{~mm}$ embedded image on the $12 \times 9 \mathrm{~mm}$ en face image indicates the location of the OCTA en face images. (B) Early Treatment Diabetic Retinopathy Study contour, macular vessel density maps of the (C) SCP and (D) DCP. (C and D) Mild changes in vessel density, indicating the disappearance of certain blood vessels around the fovea in the SCP and DCP. (E) Mild enlargement of the foveal avascular zone. (F) SCP segmentation and (G) DCP segmentation from a B-scan of OCT combined with vessel signals. SCP, superficial capillary plexus; DCP, deep capillary plexus; OCTA, optical coherence tomography angiography.

after SOR. However, the parafoveal, temporal, superior and nasal inner retinal thickness decreased during the SOT period.

Silicone oil has been commonly used to treat complex cases of vitreoretinal disease. However, opinions on the retinal effects of silicone oil are inconsistent. A previous study of 500 patients indicated no evidence of toxicity to the retina associated with the use of silicone oil, even in a number of cases that were monitored for up to 8 years (14). However, Ma et al (15) reported that for the use of silicone oil for periods of $<9$ months, silicone oil vesicles were observed only on the surface of the retina, whereas after 9 months, these vesicles began to enter the sensory layer of the retina. Furthermore, the use of SOT for $>9$ months causes alterations to retinal saturation and narrowing of retinal arterioles, which may further interfere with oxygen metabolism in the retina; no such changes were observed within a tamponade period of $\leq 9$ months (16).

In addition, to the best of our knowledge, no previous studies have addressed the effects of silicone oil on retinal vessel density in patients. In the present study, a non-invasive OCTA technique was adopted to detect macular capillary changes in the eyes of patients with SOT, and no obvious changes in 
Table II. Vessel density of SCP, DCP and area of FAZ in eyes with silicone oil tamponade.

\begin{tabular}{lccc}
\hline Item & 1st follow-up & 2nd follow-up & P-value \\
\hline $\begin{array}{l}\text { Vessel density } \\
\text { of SCP }\end{array}$ & & & \\
Whole & $45.24 \pm 4.65$ & $43.08 \pm 6.22$ & 0.15 \\
Fovea & $24.12 \pm 6.43$ & $21.94 \pm 9.84$ & 0.15 \\
Parafovea & $47.60 \pm 5.33$ & $45.68 \pm 6.97$ & 0.24 \\
Superior-hemi & $47.68 \pm 5.55$ & $45.96 \pm 7.32$ & 0.31 \\
Inferior-hemi & $47.52 \pm 5.48$ & $45.46 \pm 6.88$ & 0.22 \\
Tempo & $46.36 \pm 5.73$ & $44.79 \pm 8.08$ & 0.43 \\
Superior & $47.88 \pm 6.28$ & $46.37 \pm 6.93$ & 0.38 \\
Nasal & $48.39 \pm 4.96$ & $45.59 \pm 7.49$ & 0.09 \\
Inferior & $47.80 \pm 6.04$ & $46.00 \pm 6.57$ & 0.27 \\
Vessel density & & & \\
of DCP & & & \\
Whole & $49.28 \pm 7.10$ & $49.87 \pm 6.80$ & 0.57 \\
Fovea & $29.15 \pm 6.44$ & $28.45 \pm 7.93$ & 0.70 \\
Parafovea & $51.66 \pm 8.11$ & $52.44 \pm 7.45$ & 0.46 \\
Superior-hemi & $52.39 \pm 7.95$ & $52.78 \pm 7.76$ & 0.76 \\
Inferior-hemi & $50.48 \pm 8.36$ & $51.67 \pm 7.18$ & 0.27 \\
Tempo & $50.61 \pm 8.53$ & $51.05 \pm 7.21$ & 0.75 \\
Superior & $52.98 \pm 8.85$ & $53.85 \pm 8.42$ & 0.56 \\
Nasal & $52.30 \pm 7.66$ & $52.66 \pm 7.65$ & 0.79 \\
Inferior & $50.75 \pm 8.98$ & $53.55 \pm 10.62$ & 0.16 \\
FAZ & $0.45 \pm 0.26$ & $0.45 \pm 0.22$ & 0.99 \\
\hline
\end{tabular}

Values are expressed as the mean \pm standard deviation. SCP, superficial capillary plexus; DCP, deep capillary plexus; FAZ, foveal avascular zone.

vessel density in SCP and DCP during SOT or following SOR were identified. This indicated that silicone oil had no effect on the retinal vasculature when used for $<6$ months, suggesting that it has no significant toxic effect on the retinal vasculature. Furthermore, the present results were consistent with those of Yang et al (17), which demonstrated that SOT in rabbit eyes may not cause any pathological changes in the retinal vascular or retinal hypoxia over a period of 6 months.

However, the present results appear to contradict those of previous studies. Kubicka-Trzaska et al (18) assessed macular microcirculatory blood flow using Doppler laser scanning to demonstrate that silicone oil may negatively impact the retinal microcirculation as early as 1 month after surgery. In addition, Effert et al (19) measured the arteriovenous passage times of silicone-oil-filled eyes using a laser scan ophthalmoscope and reported a prolongation of the arteriovenous passage time in the silicone-oil-filled eye as compared with that in the contralateral eye. The above study concluded that silicone oil may have a negative long-term effect on retinal microcirculation. However, the two studies mentioned above included a subject group with unilateral macular-on complex rhegmatogenous retinal detachment (RRD) and a set of normal contralateral eyes as the control, which were not
Table III. Retinal thickness in the silicone oil tamponade group.

\begin{tabular}{lllr}
\hline Item & 1st follow-up & 2nd follow-up & P-value \\
\hline $\begin{array}{llll}\text { Full retinal } \\
\text { thickness }\end{array}$ & & \\
$\quad$ Fovea & $229.5 \pm 50.81$ & $243.3 \pm 40.18$ & 0.16 \\
Parafovea & $296.0 \pm 34.91$ & $289.0 \pm 21.09$ & 0.28 \\
Superior-hemi & $294.4 \pm 30.17$ & $286.7 \pm 22.06$ & 0.14 \\
Inferior-hemi & $297.7 \pm 40.48$ & $291.3 \pm 21.33$ & 0.42 \\
Tempo & $280.3 \pm 29.51$ & $278.0 \pm 25.19$ & 0.63 \\
Superior & $298.0 \pm 30.27$ & $289.1 \pm 21.65$ & 0.11 \\
Nasal & $299.0 \pm 46.16$ & $294.0 \pm 24.26$ & 0.56 \\
Inferior & $302.3 \pm 44.68$ & $294.6 \pm 20.19$ & 0.40 \\
Inner retinal & & & \\
thickness & & & \\
Fovea & $67.09 \pm 18.81$ & $67.04 \pm 15.78$ & 0.99 \\
Parafovea & $110.8 \pm 10.51$ & $105.1 \pm 8.83$ & 0.01 \\
Superior-hemi & $111.5 \pm 12.27$ & $104.8 \pm 11.41$ & 0.01 \\
Inferior-hemi & $110.2 \pm 9.64$ & $118.3 \pm 62.95$ & 0.54 \\
Tempo & $107.0 \pm 11.99$ & $100.7 \pm 10.92$ & 0.02 \\
Superior & $112.8 \pm 13.39$ & $106.8 \pm 12.73$ & 0.03 \\
Nasal & $112.8 \pm 13.52$ & $105.4 \pm 12.01$ & $<0.01$ \\
Inferior & $110.8 \pm 9.89$ & $107.6 \pm 6.67$ & 0.18 \\
\hline & & & \\
\hline
\end{tabular}

Values are expressed as the mean \pm standard deviation.

directly comparable. As RRD itself may damage the retinal microcirculation, it was unreasonable to suggest that silicone oil directly caused a reduction in the macular microcirculation. In addition, the study focused on retinal blood flow but not vessel density.

It is known that silicone oil has lower specific gravity than the aqueous phase of the vitreous; thus, silicone oil exerts pressure on the retina in the sitting, lateral and prone positions. Whether this effect affects retinal thickness in the long-term has so far remained elusive. The present results demonstrated that, the inferior inner retinal thickness remained unchanged, whilst the parafoveal, temporal, superior and nasal inner retinal thickness were decreased in eyes with SOT, which implies that silicone oil may compress the inner retina and decrease the inner retinal thickness. However, this requires further investigation.

As the present study had a retrospective design, not all patients regularly visited our hospital following surgery. Thus, the present study was limited to a relatively small number of subjects. Therefore, 23 patients in the SOT group and 20 in the SOR group were assessed separately, rather than as a single group of patients who underwent SOT and subsequent SOR. However, further prospective studies with a larger sample size should be performed.

In conclusion, the present results demonstrate that the use of silicone oil for $<6$ months has no significant effect on macular capillary vessel density. However, reduced inner retinal thickness in eyes with SOT was also demonstrated. Therefore, it is recommended that silicone oil is removed as soon as possible once the ocular disease is stable. Whether silicone oil 
Table IV. Vessel density SCP, DCP and area of FAZ in the eyes with silicone oil removal.

\begin{tabular}{|c|c|c|c|}
\hline Item & Pre-Op & Post-Op & P-value \\
\hline \multicolumn{4}{|l|}{$\begin{array}{l}\text { Vessel density } \\
\text { of SCP }\end{array}$} \\
\hline Whole & $41.89 \pm 4.33$ & $41.88 \pm 4.30$ & 0.99 \\
\hline Fovea & $23.68 \pm 8.57$ & $27.28 \pm 7.63$ & 0.18 \\
\hline Parafovea & $43.66 \pm 5.03$ & $43.46 \pm 4.50$ & 0.83 \\
\hline Superior-hemi & $44.40 \pm 4.98$ & $44.09 \pm 4.65$ & 0.76 \\
\hline Inferior-hemi & $42.98 \pm 5.43$ & $42.85 \pm 4.82$ & 0.89 \\
\hline Tempo & $42.81 \pm 6.26$ & $42.15 \pm 5.55$ & 0.55 \\
\hline Superior & $44.83 \pm 5.12$ & $44.97 \pm 4.90$ & 0.90 \\
\hline Nasal & $43.90 \pm 5.48$ & $43.78 \pm 4.42$ & 0.91 \\
\hline Inferior & $43.20 \pm 5.96$ & $42.95 \pm 5.73$ & 0.80 \\
\hline \multicolumn{4}{|l|}{$\begin{array}{l}\text { Vessel density } \\
\text { of DCP }\end{array}$} \\
\hline Whole & $47.21 \pm 6.48$ & $48.18 \pm 5.92$ & 0.44 \\
\hline Fovea & $32.31 \pm 9.40$ & $34.93 \pm 11.68$ & 0.40 \\
\hline Parafovea & $48.80 \pm 7.35$ & $49.92 \pm 7.35$ & 0.40 \\
\hline Superior-hemi & $47.99 \pm 8.39$ & $50.15 \pm 7.91$ & 0.18 \\
\hline Inferior-hemi & $49.12 \pm 6.45$ & $48.68 \pm 6.10$ & 0.73 \\
\hline Tempo & $48.71 \pm 7.33$ & $48.14 \pm 8.56$ & 0.71 \\
\hline Superior & $48.35 \pm 9.57$ & $51.23 \pm 7.90$ & 0.16 \\
\hline Nasal & $48.53 \pm 7.50$ & $48.71 \pm 8.84$ & 0.93 \\
\hline Inferior & $49.57 \pm 8.17$ & $50.07 \pm 7.84$ & 0.74 \\
\hline FAZ & $0.63 \pm 0.49$ & $0.85 \pm 1.23$ & 0.41 \\
\hline
\end{tabular}

Values are expressed as the mean \pm standard deviation. SCP, superficial capillary plexus; DCP, deep capillary plexus; FAZ, foveal avascular zone; OP, operation.

irreversibly affects retinal thickness requires assessment in a prospective study with an increased sample size.

\section{Acknowledgements}

Not applicable.

\section{Funding}

The current study was supported by the National Natural Science Foundation of China (grant no. 81700817).

\section{Availability of data and materials}

The datasets used and/or analyzed in the present study are available from the corresponding author on reasonable request.

\section{Authors' contributions}

WX drafted the manuscript. ZZ, LZ and RL acquired the data. SZ was responsible for the treatment of the patients. WX, YW and WC analyzed the data and revised the manuscript. All authors read and approved the final manuscript.
Table V. Retinal thickness in eyes with silicone oil removal.

\begin{tabular}{lccc}
\hline Item & Pre-Op & Post-Op & P-value \\
\hline Full retinal & & & \\
thickness & & & \\
$\quad$ Fovea & $245.0 \pm 47.53$ & $247.3 \pm 50.92$ & 0.70 \\
Parafovea & $302.7 \pm 43.19$ & $308.3 \pm 44.26$ & 0.15 \\
Superior-hemi & $315.9 \pm 72.99$ & $320.9 \pm 71.75$ & 0.33 \\
Inferior-hemi & $289.3 \pm 31.34$ & $295.6 \pm 37.46$ & 0.07 \\
Tempo & $280.3 \pm 50.14$ & $286.5 \pm 53.52$ & 0.11 \\
Superior & $333.5 \pm 89.96$ & $334.3 \pm 82.47$ & 0.90 \\
Nasal & $311.8 \pm 55.77$ & $318.9 \pm 50.78$ & 0.18 \\
Inferior & $289.7 \pm 35.83$ & $295.7 \pm 41.43$ & 0.08 \\
Inner retinal & & & \\
thickness & & & \\
Fovea & $71.85 \pm 21.50$ & $72.60 \pm 19.62$ & 0.88 \\
Parafovea & $103.0 \pm 14.13$ & $105.5 \pm 17.25$ & 0.40 \\
Superior-hemi & $104.6 \pm 15.83$ & $107.8 \pm 18.26$ & 0.38 \\
Inferior-hemi & $101.4 \pm 15.89$ & $103.5 \pm 17.83$ & 0.48 \\
Tempo & $96.15 \pm 21.12$ & $98.80 \pm 20.36$ & 0.35 \\
Superior & $106.5 \pm 17.98$ & $109.7 \pm 20.42$ & 0.44 \\
Nasal & $107.0 \pm 16.42$ & $109.9 \pm 18.69$ & 0.46 \\
Inferior & $102.2 \pm 17.61$ & $103.9 \pm 19.83$ & 0.61 \\
\hline & & & \\
\hline
\end{tabular}

Values are expressed as the mean \pm standard deviation. OP, operation.

\section{Ethics approval and consent to participate}

The study was approved by the Ethics Committee of Zhongshan Ophthalmic Center (Guangzhou, China). Informed consent was obtained from each participant.

\section{Patient consent for publication}

Not applicable.

\section{Competing interests}

The authors declare that they have no competing interests.

\section{References}

1. Cibis PA, Becker B, Okun E and Canaan S: The use of liquid silicone in retinal detachment surgery. Arch Ophthalmol 68: 590-599, 1962.

2. Pastor JC: Proliferative vitreoretinopathy: An overview. Surv Ophthalmol 43: 3-18, 1998.

3. Azen SP, Scott IU, Flynn HJ Jr, Lai MY, Topping TM, Benati L, Trask DK and Rogus LA: Silicone oil in the repair of complex retinal detachments. A prospective observational multicenter study. Ophthalmology 105: 1587-1597, 1998.

4. Ortisi E, Avitabile T and Bonfiglio V: Surgical management of retinal detachment because of macular hole in highly myopic eyes. Retina 32: 1704-1718, 2012.

5. Wei Y, Li Y and Chen F: Vitrectomy treatment of retinal detachments related to choroidal coloboma involving the disk. Retina 34: 1091-1095, 2014.

6. Kapur R, Birnbaum AD, Goldstein DA, Tessler HH, Shapiro MJ, Ulanski LJ and Blair MP: Treating uveitis-associated hypotony with pars plana vitrectomy and silicone oil injection. Retina 30: 140-145, 2010. 
7. Toklu Y, Cakmak HB, Ergun SB, Yorgun MA and Simsek S: Time course of silicone oil emulsification. Retina 32: 2039-2044, 2012.

8. Kampik A and Gandorfer A: Silicone oil removal strategies. Semin Ophthalmol 15: 88-91, 2000.

9. Jia Y, Morrison JC, Tokayer J, Tan O, Lombardi L, Baumann B, Lu CD, Choi W, Fujimoto JG and Huang D: Quantitative OCT angiography of optic nerve head blood flow. Biomed Opt Express 3: 3127-3137, 2012

10. Jia Y, Bailey ST, Wilson DJ, Tan O, Klein ML, Flaxel CJ, Potsaid B, Liu JJ, Lu CD, Kraus MF, et al: Quantitative optical coherence tomography angiography of choroidal neovascularization in age-related macular degeneration. Ophthalmology 21: $1435-1444,2014$

11. Jia Y, Tan O, Tokayer J, Potsaid B, Wang Y, Liu JJ, Kraus MF, Subhash H, Fujimoto JG, Hornegger J and Huang D: Split-spectrum amplitude-decorrelation angiography with optical coherence tomography. Opt Express 20: 4710-4725, 2012

12. Koustenis A Jr, Harris A, Gross J, Januleviciene I, Shah A and Siesky B: Optical coherence tomography angiography: An overview of the technology and an assessment of applications for clinical research. Brit J Ophthalmol 101: 16-20, 2017.
13. Yu J, Xiao K, Huang J, Sun X and Jiang C: Reduced retinal vessel density in obstructive sleep apnea syndrome patients: An optical coherence tomography angiography study. Invest Ophth Vis Sci 58: 3506-3512, 2017.

14. Lucke $\mathrm{K}$ and Laqua H: Visual outcome after silicon oil surgery Fortschr Ophthalmol 88: 603-607, 1991 (In German).

15. Gisbert R, Ulrich S and Alexander AB: Pathological changes of occular tissue after solicone oil filling. Chin J Ocular Fundus Dis: 35-37, 1999.

16. Lou B, Yuan Z, He L, Lin L, Gao Q and Lin X: The changes of retinal saturation after long-term tamponade with silicone oil. Biomed Res Int 2015: 713828, 2015.

17. Yang W, Yuan Y, Zong Y, Huang Z, Mai S, Li Y, Qian X, Liu Y and Gao Q: Preliminary study on retinal vascular and oxygen-related changes after long-term silicone oil and foldable capsular vitreous body tamponade. Sci Rep 4: 5272, 2014.

18. Kubicka-Trzaska A, Kobylarz J and Romanowska-Dixon B: Macular microcirculation blood flow after pars plana vitrectomy with silicone oil tamponade. Klin Oczna 113: 146-148, 2011.

19. Effert R, Wolf S, Arend O, Schulte K and Reim M: Retinal hemodynamics after pars plana vitrectomy with silicone oil tamponade. Ger J Ophthalmol 3: 65-67, 1994. 